OPEN ACCESS

Edited by: Jie Yin,

Institute of Subtropical Agriculture

(CAS), China

Reviewed by:

Yun-wei Dong,

Xiamen University, China

Tomoo Sawabe,

Hokkaido University, Japan

${ }^{*}$ Correspondence:

Lina Sun

sunlina@qdio.ac.cn

Hongsheng Yang

hshyang@qdio.ac.cn

Specialty section:

This article was submitted to

Microbial Symbioses,

a section of the journal

Frontiers in Microbiology

Received: 11 March 2019

Accepted: 07 May 2019

Published: 04 June 2019

Citation:

Zhang H, Wang Q, Liu S, Huo D,

Zhao J, Zhang L, Zhao Y, Sun L and Yang $H$ (2019) Genomic

and Metagenomic Insights Into

the Microbial Community

in the Regenerating Intestine of the

Sea Cucumber Apostichopus japonicus. Front. Microbiol. 10:1165.

doi: 10.3389/fmicb.2019.01165

\section{Genomic and Metagenomic Insights Into the Microbial Community in the Regenerating Intestine of the Sea Cucumber Apostichopus japonicus}

\author{
Hongxia Zhang 1,2,3, Qing Wang ${ }^{3,4}$, Shilin Liu'1,2,3, Da Huo 1,2,3,5, Jianmin Zhao ${ }^{3,4}$, \\ Libin Zhang ${ }^{1,2,3,5}$, Ye Zhao ${ }^{6}$, Lina Sun ${ }^{1,2,3,5 *}$ and Hongsheng Yang ${ }^{1,2,3,5 *}$ \\ ${ }^{1}$ CAS Key Laboratory of Marine Ecology and Environmental Sciences, Institute of Oceanology, Chinese Academy of \\ Sciences (CAS), Qingdao, China, ${ }^{2}$ Laboratory for Marine Ecology and Environmental Science, Qingdao National Laboratory \\ for Marine Science and Technology, Qingdao, China, ${ }^{3}$ Center for Ocean Mega-Science, Chinese Academy of Sciences \\ (CAS), Qingdao, China, ${ }^{4}$ CAS Key Laboratory of Coastal Environmental Processes and Ecological Remediation, Yantai \\ Institute of Coastal Zone Research, Chinese Academy of Sciences (CAS), Yantai, China, ${ }^{5}$ University of Chinese Academy of \\ Sciences, Beijing, China, ${ }^{6}$ Ocean School, Yantai University, Yantai, China
}

Host-intestine microbiota interactions have been widely studied in aquatic animals, but these interactions in the intestine regeneration process of the sea cucumber Apostichopus japonicus have been rarely investigated. To understand how intestine regeneration impacts the developing intestinal microbiome composition and function, we performed a case study to characterize the intestinal microbial composition and functional genes of $A$. japonicus during intestine regeneration stages. Highthroughput 16S rRNA gene sequencing revealed significantly different intestine microbiota compositions in different regeneration stages. The phylogenetic diversity and composition of the intestinal microbiota changed significantly in the early regeneration stage and tended to recover in the end stage. During the regeneration process, the abundance of Bacteroidetes and Rhodobacterales increased significantly. A network analysis revealed that Rhodobacteraceae and Flavobacteriaceae may function as keystone taxa in the intestinal microbial community of $A$. japonicus during intestine regeneration. Metagenomic analyses of representative samples revealed that the microbiomes of regenerating intestines were enriched in genes facilitating cell proliferation, digestion and immunity. The increased abundance of Bacteroidetes elevated the enrichment of genes associated with carbohydrate utilization. Some functional features in the subsystem category changed in a pattern that was consistent with the changing pattern of microbiota composition during intestine regeneration. Our results revealed that seemingly regular alterations in the intestinal microbiome composition and function are associated with intestine regeneration stages. Intestinal microbiota can increase the abundance of beneficial bacterial members and upregulate related functional genes to adapt to intestine regeneration and reconstruct a stable community structure. This study provides a new insight into the mechanism of the host-microbiota interaction response to organ regeneration.

Keywords: Apostichopus japonicus, intestine regeneration, intestinal microbiota, genomic, metagenomic 


\section{INTRODUCTION}

Intestinal microbiome, which exert important influences on a host's development and tissue physiology, immune regulation, metabolic absorption and so on, have been widely studied in many organisms. For instance, intestinal bacteria can enhance the stability of $\beta$-catenin in intestinal epithelial cells and promote rates of cell proliferation in the vertebrate intestine (Cheesman et al., 2011). A previous study also found that different types of intestinal microbiota enhanced the healing of intestinal anastomoses in mice (Okada et al., 1999). In addition, recent research has revealed that the intestinal microbiome modulates animal behaviors, and shapes various aspects of basic neurodevelopmental processes, such as the formation of the blood-brain barrier, neurogenesis, myelination, and microglia maturation (Sharon et al., 2016).

The sea cucumber Apostichopus japonicus is an economically important fishery resource along the East Asian coast. Sea cucumbers possesses high nutritional and economic value as they are abundant in protein and mucopolysaccharides, and their market demand is high; therefore, the scale of farming is growing rapidly. A. japonicus ingests organic matter, microbes, algae, protozoa as well as aquatic animal detritus and thus affects the benthic material recycling (Choo, 2008; Yamazaki et al., 2016). The aforementioned broad-spectrum characteristics of intestinal microbiota define them as integral contributors to the development, growth and physiological health of the sea cucumber A. japonicus. In the past decade, studies have focused on (i) bacterial community structures in the intestine of A. japonicus (Gao et al., 2014a,b); (ii) the physiological characterization of intestinal bacterial isolates (Zhang et al., 2013; Wang et al., 2015); and (iii) the potential effects of intestinal bacteria on sea cucumber growth. For instance, studies report that larger and smaller A. japonicus individuals have significantly different intestinal microbial communities (Sha et al., 2016; Yamazaki et al., 2016). Recent studies have attempted to explain how intestinal microbiota are shaped by external factors, such as probiotics, diet and habitat types (Yang et al., 2015; Qi et al., 2017; Wang et al., 2017; Zhao et al., 2018; Ma et al., 2019). However, internal factors of organisms that may contribute to intestinal microbiota modulation have been scarcely explored.

Sea cucumbers are an excellent model to study organ regeneration since they possess a unique biological mechanism called evisceration when they are subjected to natural or induced stimulation (Dolmatov and Ginanova, 2009; Li et al., 2017b; Sun et al., 2017a). The lost organs, including the intestines, hemal system and respiratory trees, can concurrently regenerate within a few weeks (Shukalyuk and Dolmatov, 2001; Sun et al., 2011). Regeneration is a fascinating biological event that has aroused interest in many researchers. For the past two decades, studies have investigated many mechanisms of regenerative processes in sea cucumbers, including morphological features, cell division, dedifferentiation, cell proliferation and migration, nerve regrowth, and molecular regulatory mechanisms (GarcíaArrarás et al., 1998; Dolmatov and Ginanova, 2009; Sun et al., 2011, 2017a, 2018; Sun L.N. et al., 2013; Li et al., 2017b; Zhang et al., 2017). Moreover, the intestine regeneration process is accompanied by the reconstruction of the intestinal microbial community. To the best of our knowledge, only one study thus far has addressed the question of variation in the intestinal bacterial composition of $A$. japonicus during intestine regeneration stages (Wang et al., 2018). This study found that the bacterial community structure in intestine of $A$. japonicus varied greatly in different regeneration stages, with a significant difference between the earlier and later stages. Therefore, little is known about how such life events impact the development of the intestinal microbiome composition and function.

Previous studies have shown that hosts can develop tolerance to intestinal microbial antigens early in life and then maintain a mutually beneficial relationship with intestinal microbiota (Knoop et al., 2017). Adult intestinal microbiota are thought to be relatively stable over time, and this stability imparts resilience to various perturbations, ensuring the host's continued intestinal function (Koenig et al., 2011). Therefore, we are interested in how the intestinal microbiota recover such stability and resilience. Understanding the regulatory mechanisms of microbial associations in the regenerating intestine may help develop a theoretical basis for further understanding the role of intestinal microbiome in the process of tissue and organ regeneration.

We analyzed the compositions and functional characteristics of microbial community in the regenerating intestine of A. japonicus using 16S rRNA gene and metagenomic sequencing. These results were used to address the following questions: How does the intestinal microbiome composition and function respond to intestinal regeneration? What are the keystone taxa in the intestinal microbial community of A. japonicus during intestine regeneration? This analysis allows us to pinpoint specific events (intestine regeneration) likely associated with significant changes in the intestinal microbiome, which is of great theoretical significance to the healthy aquaculture of sea cucumbers and to the understanding of host-gut microbiota interactions.

\section{MATERIALS AND METHODS}

\section{Experimental Animals and Sample Collection}

Adult A. japonicas (of 80-100 g) were collected in October 2017 from the coast of Weihai, Shandong, China. The animals were acclimated in seawater at $15 \pm 1^{\circ} \mathrm{C}$ for 2 weeks prior to treatment and were fed a formulated diet once a day. After acclimation, evisceration was induced by injecting approximately $2 \mathrm{~mL} 0.35$ M KCl into the coelomic cavity (Sun L. et al., 2013; Sun et al., 2017b). Eviscerated animals were kept in well-aerated indoor seawater tanks. We began recording observations at the point when the sea cucumber had expelled the entire intestine. At least 9 individuals (three biological replicates $\times$ three individuals per biological replicate) per regeneration stage $[10,14,18$, and 21 days postevisceration (dpe), labeled as D10, D14, D18, and D21, respectively] were used for analyses. Sea cucumbers that did not undergo evisceration were used as the control (three biological replicates, labeled as CK). After 7 days of intestine regeneration, lumen formation of the new intestine began; the 
intestine gradually developed to form a complete structure in which the digestive and absorptive functions were restored during days 14-21 of intestine regeneration (Sun et al., 2011; Sun L. et al., 2013). The 10th day was therefore an appropriate time point for sampling the new intestine.

For intestine collection, 9 A. japonicus individuals were randomly sampled in different regeneration stages. Individuals were rinsed with sterile seawater and moved into sterile plates. The coelomic fluid was withdrawn from the coelom of sea cucumbers using sterile syringes. The intestines were then aseptically dissected and transferred into sterile tubes, and preserved at $-80^{\circ} \mathrm{C}$ until DNA extraction.

\section{DNA Extraction and 16S rRNA Gene Sequencing}

Total DNA was extracted from the gut contents of $A$. japonicas by using a FastDNA SPIN Kit for Feces (MP Biomedicals, Santa Ana, CA, United States) in accordance with the instructions provided by the manufacturer. The extracted DNA was dissolved in $50 \mu \mathrm{L}$ of TE buffer, quantified using a NanoDrop spectrophotometer (NanoDrop, Thermo Fisher Scientific, United States) and stored at $-20^{\circ} \mathrm{C}$ prior to analysis. PCR amplification of bacterial $16 \mathrm{~S}$ rRNA hypervariable regions V4-V5 was conducted using the universal primer set 515f (GTGCCAGCMGCCGCGGTAA) and 907r (CCGTCAATTCMTTTRAGTTT), with 5 bp barcodes fused to the forward primer to allow sample multiplexing. The purified PCR products with different barcodes were normalized in equimolar amounts and then prepared using an NEB Next ${ }^{\circledR}$ Ultra ${ }^{\mathrm{TM}}$ DNA Library Prep Kit for Illumina (NEB, United States) following the manufacturer's protocol and sequenced on an Illumina HiSeq platform.

\section{Deep Sequencing Data Processing}

Raw deep sequencing data were processed using Quantitative Insights Into Microbial Ecology software (QIIME version 1.9.01; Caporaso et al., 2010) with the default parameters unless otherwise noted. After all chimeric and low-quality reads were removed, qualified sequences were clustered into operational taxonomic units (OTUs) at the 97\% identity threshold level, and the most abundant sequence from each OTU was chosen as a representative sequence for that OTU. Taxonomic classification of each OTU was assigned using the Ribosomal Database Project classifier. The average relative abundance (\%) of predominant genus-level taxonomic groups in each sample was estimated by comparing the number of sequences assigned to a specific taxon vs. the number of total sequences obtained for that sample. Based on the OTU numbers, principal components analysis (PCA) was performed using $\mathrm{R}$ version 3.0 .2 (package stats) to test the differences in microbial community structures.

\section{Metagenomic Sequencing}

Each representative DNA sample from the sea cucumber intestine for which sufficient volumes were available after 16S typing was used for metagenomic sequencing. Metagenomic DNA pairedend libraries were prepared with an insert size of $350 \mathrm{bp}$ and

${ }^{1}$ http://www.qiime.org were quantified using a Qubit Fluorometer (Life Technologies, Carlsbad, CA, United States) and Agilent 2100 bioanalyzer (Agilent Technologies, Palo Alto, CA, United States). Sequencing was performed on an Illumina HiSeq PE150 platform.

Raw reads were preprocessed using FasqMcf to exclude adapter sequences and low-quality sequences (Aronesty, 2011), and reads derived from host contamination were filtered using bowtie 2.2.4 (Langmead and Salzberg, 2012) with the parameters “-end-to-end, -sensitive, -I 200, -X 400." Clean reads were assembled and analyzed by SOAPdenovo software (V2.04) (Luo et al., 2012) using the following parameters “-d 1, -M 3, -R, $-\mathrm{u},-\mathrm{F},-\mathrm{K}$ 55." Then, gene prediction was performed on contigs larger than 500 bp by MetaGeneMark software with the default parameter, and gene models with CDS lengths less than $100 \mathrm{bp}$ were filtered out (Qin et al., 2010; Nielsen et al., 2014). The gene catalog was constructed using the gene models predicted from each sample by CD-HIT-EST (version 4.6.6) (Li and Godzik, 2006) with the parameter “-c 0.95 -n 10 -G 0 -aS 0.9," which adopts a greedy incremental clustering algorithm and criteria as identity $>95 \%$ and overlap $>90 \%$ of the shorter genes.

\section{Metagenomic Analysis}

DIAMOND software (version 0.9.9) (Buchfink et al., 2015) was used to align the unigenes to the sequences of bacteria, fungi, archaea and viruses, which were all extracted from the NCBI nr database (Version: 2018-01-02) with the parameter “-e 1e-5." The functional assignments of protein sequences were made on the basis of DIAMOND alignment against the KEGG protein database (version 2018-01-01) (Minoru et al., 2006), eggNOG database (version 4.5) (Sean et al., 2014), and CAZy database (Version 20150704) (Cantarel et al., 2009) by using the best hit with an $e<1 \mathrm{e}-5$. For each sequence's BLAST result, the best BLAST hit was used for subsequent analysis.

Phylum, class, order, family, genus, species, KEGG orthology (KO), and orthologous group (OG) relative abundances were calculated by summing the abundance of the respective genes belonging to each category per sample based on the taxonomic assignments, $\mathrm{KO}$ and $\mathrm{OG}$ annotations, respectively. The relative gene abundance profile was also summarized into KEGG, eggNOG and CAZy functional profiles for the functional analysis.

\section{Accession Numbers of Nucleotide Sequences}

All 16S rRNA gene and metagenomic sequence raw data were submitted to the sequence read archive (SRA) with project accession numbers PRJNA512056 and PRJNA518164.

\section{RESULTS}

\section{S rRNA Gene Analysis Revealed a Temporal Pattern of Bacterial Diversity}

At $97 \%$ sequence identity, a total of 1,671 OTUs were obtained across all samples. The samples from the CK group contained 766 OTUs (on average), while D10, D14, D18, and D21 regeneration samples contained 142, 920, 916, 914 OTUs (on average), 
TABLE 1 | Summary of the alpha diversity of bacterial communities in samples from different regeneration stages.

\begin{tabular}{|c|c|c|c|c|c|}
\hline Sample ID & OTU & Chao 1 & Coverage & Shannon & Simpson \\
\hline CK & 766 & $1275(1143,1454)$ & 0.992879 & $2.95(2.93,2.97)$ & $0.1713(0.1686,0.1741)$ \\
\hline D10 & 142 & $195(163,272)$ & 0.998928 & $2.22(2.2,2.24)$ & $0.2873(0.2819,0.2927)$ \\
\hline D14 & 920 & $1181(1109,1280)$ & 0.992959 & $4.47(4.45,4.49)$ & $0.0316(0.0309,0.0322)$ \\
\hline D18 & 916 & $1205(1129,1309)$ & 0.994295 & $4.09(4.07,4.11)$ & $0.0519(0.051,0.0528)$ \\
\hline D21 & 914 & $1312(1208,1452)$ & 0.991934 & $4.06(4.04,4.09)$ & $0.0817(0.0796,0.0839)$ \\
\hline
\end{tabular}

respectively (Table $\mathbf{1}$ ). We analyzed both shared and unique OTUs in the bacterial communities of $A$. japonicus intestines during regeneration stages to further identify the dominant microbiota. Samples from different regeneration stages shared only 14 OTUs (Supplementary Figure 1 and Supplementary Table 1). Lower values for community richness were observed in the early regeneration stage (D10).

The alpha diversity of the bacterial community varied greatly in different regeneration stages. Shannon indices varied from 2.22 to 4.47, and Chaol richness ranged from 195 to 1,312 OTUs in all samples (Table 1). The earliest stage (D10) had the lowest Shannon index and Chaol richness, with 2.22 and 195 OTUs, respectively; however, the diversity and richness gradually increased in later stages. These results indicated that there were significant differences in alpha diversity of bacterial community between different regeneration stages.

\section{Characterization of the Microbiome Composition in the Intestine of Apostichopus japonicus During Different Regeneration Stages}

The bacterial communities in the intestine of sea cucumbers were classified as $\mathrm{CK}$ or regeneration stages based on a principal component analysis (PCA) of OTUs, which indicated that there were differences in bacterial community compositions. Figure 1 shows that 3 replicates from the same stage grouped together, and the bacterial community structures in different stages exhibited temporal patterns distinct from one other. Samples from the later stage (D21) close to the $\mathrm{CK}$ and other samples from different stages showed distinct separation, suggesting that the composition patterns of bacterial communities are associated with intestine regeneration stages.

Analysis of bacterial communities revealed distinct taxonomic compositions between intestine samples obtained from different regeneration stages (CK, D10, D14, D18, and D21) (Figure 2). In CK samples, Proteobacteria, Bacteroidetes and Firmicutes phyla predominated (96.2\% of total OTU), as was also the case in D21 samples (Supplementary Figure 2). Most of the Proteobacteria reads were assigned as Gammaproteobacteria (average 78.9\%), of which Vibrio was the dominant genus (71.5\%). Flavobacteriia (7.7\%), Bacilli (3.1\%) and Alphaproteobacteria (2.3\%) were the next most abundant classes. Flavobacteriia-related reads were predominantly affiliated with the genus Lutibacter (7.4\%).

In the D10 samples, the Proteobacteria phylum (average 97.1\%) was represented mostly by the Gammaproteobacteria $(81.8 \%)$, Alphaproteobacteria (8.7\%), and Betaproteobacteria

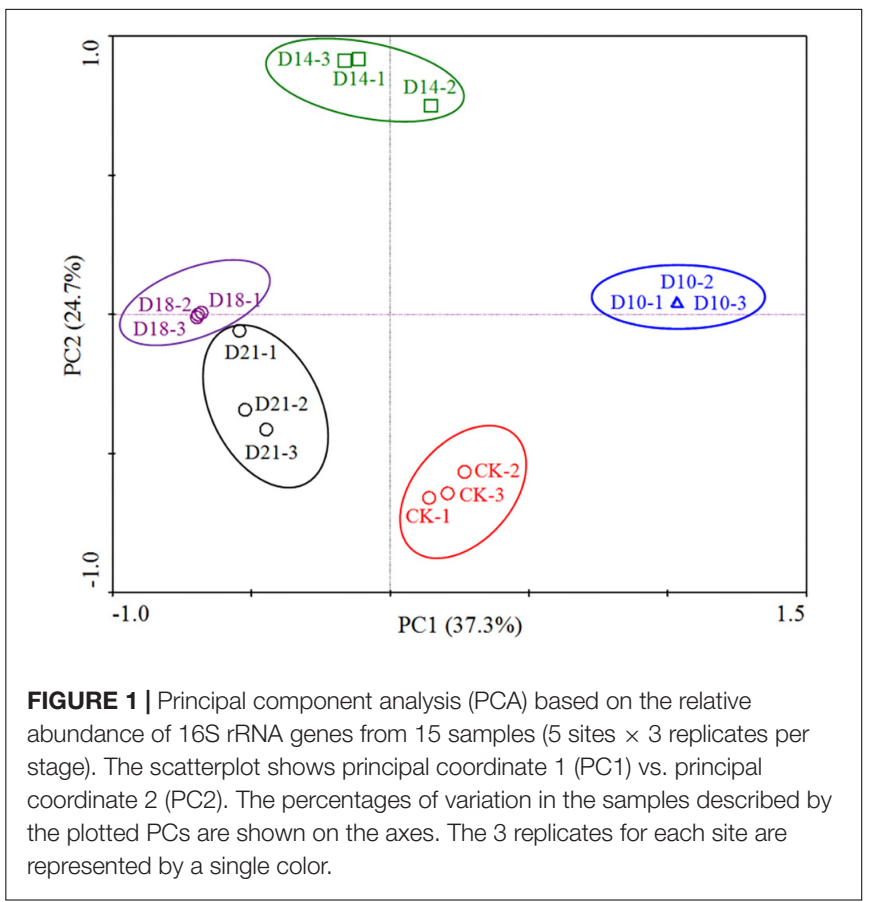

(6.6\%) classes, mainly related to Halomonas (10.7\%), Ralstonia (6.4\%), and Pelagibacterium (5.1\%) genera. In the D14 samples, the Proteobacteria phylum (average 66.4\%) was represented mostly by the Gammaproteobacteria (43.3\%), Betaproteobacteria (12.2\%), and Alphaproteobacteria (10.0\%) classes, with Pseudoalteromonas, Colwellia, Ralstonia, and Vibrio comprising $14.2,10.3,8.2$, and $6.9 \%$ of the total, respectively. Bacteroidetes (average 22.3\%) was the next most abundant phylum, represented mainly by the Sphingobacteriia (11.5\%) and Flavobacteriia (10.7\%) classes and mainly related to the Sphingobacteriales_norank (10.4\%) genus.

The composition of the intestinal bacterial community varied greatly in the D18 samples at the class and genus levels. Within the Proteobacteria (64.6\%, on average) phylum, the Alphaproteobacteria (48.0\%) and Gammaproteobacteria (16.2\%) classes predominated. Of these, $18.6 \%$ of the reads were assigned as unclassified Rhodobacteraceae, $12.4 \%$ as Vibrio, and 5.5\% as Roseobacter clade NAC11-7. Bacteroidetes (average 31.9\%) were represented mainly by the Flavobacteriia (30.89\%) class and were mainly related to the Flavobacteriaceae_uncultured (11.3\%), Cellulophaga (7.5\%), and Pseudofulvibacter (6.0\%) genera.

Compared to $\mathrm{CK}$ samples, a marked recovery profile was detected in D21 samples (Figure 2 and Supplementary Figure 2). 


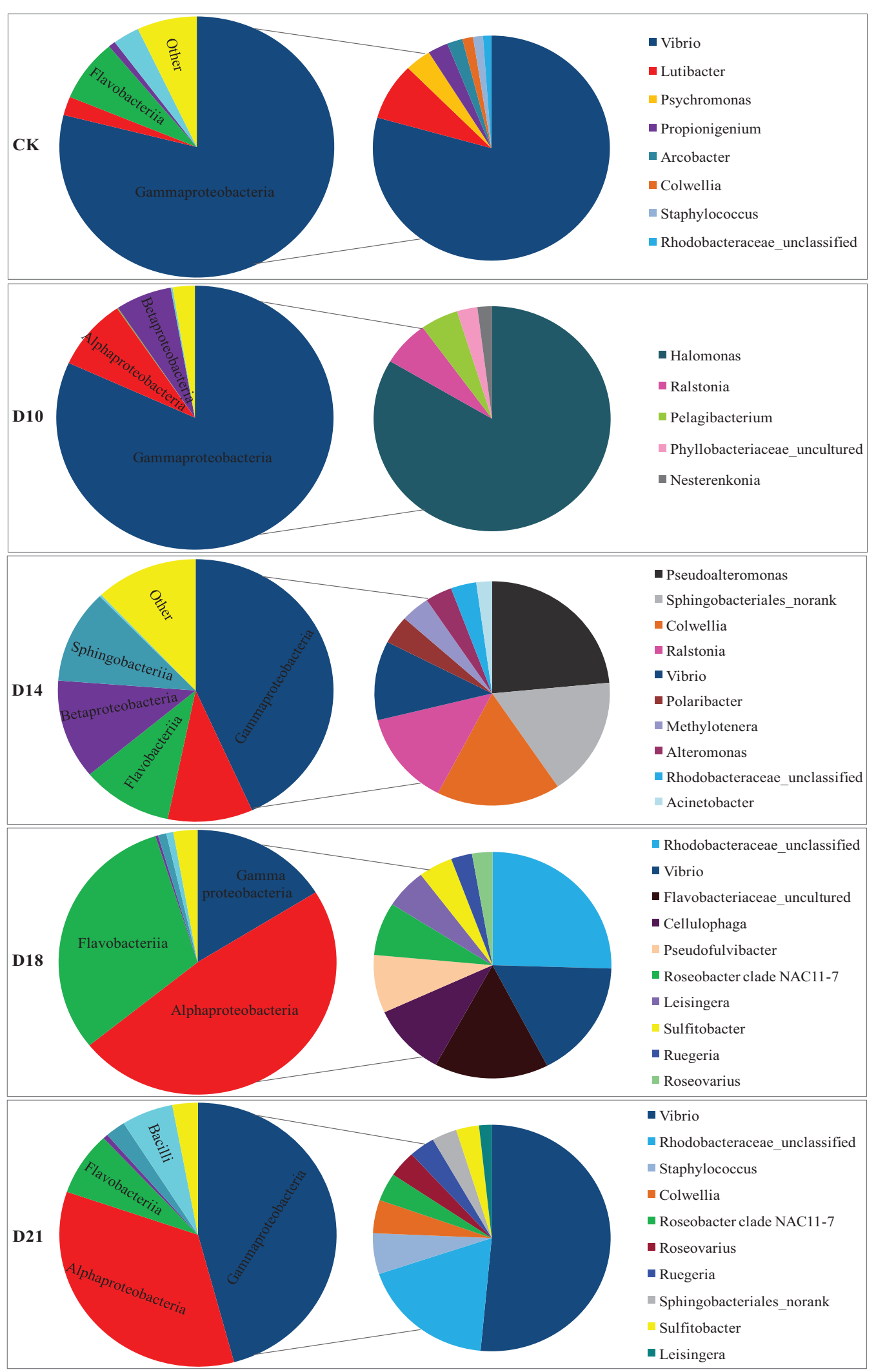

FIGURE 2 | Composition of bacterial communities in the intestine of the sea cucumber Apostichopus japonicus during different regeneration stages (\%). Note: The big pie chart is the class level, the small pie chart is the genus level. 


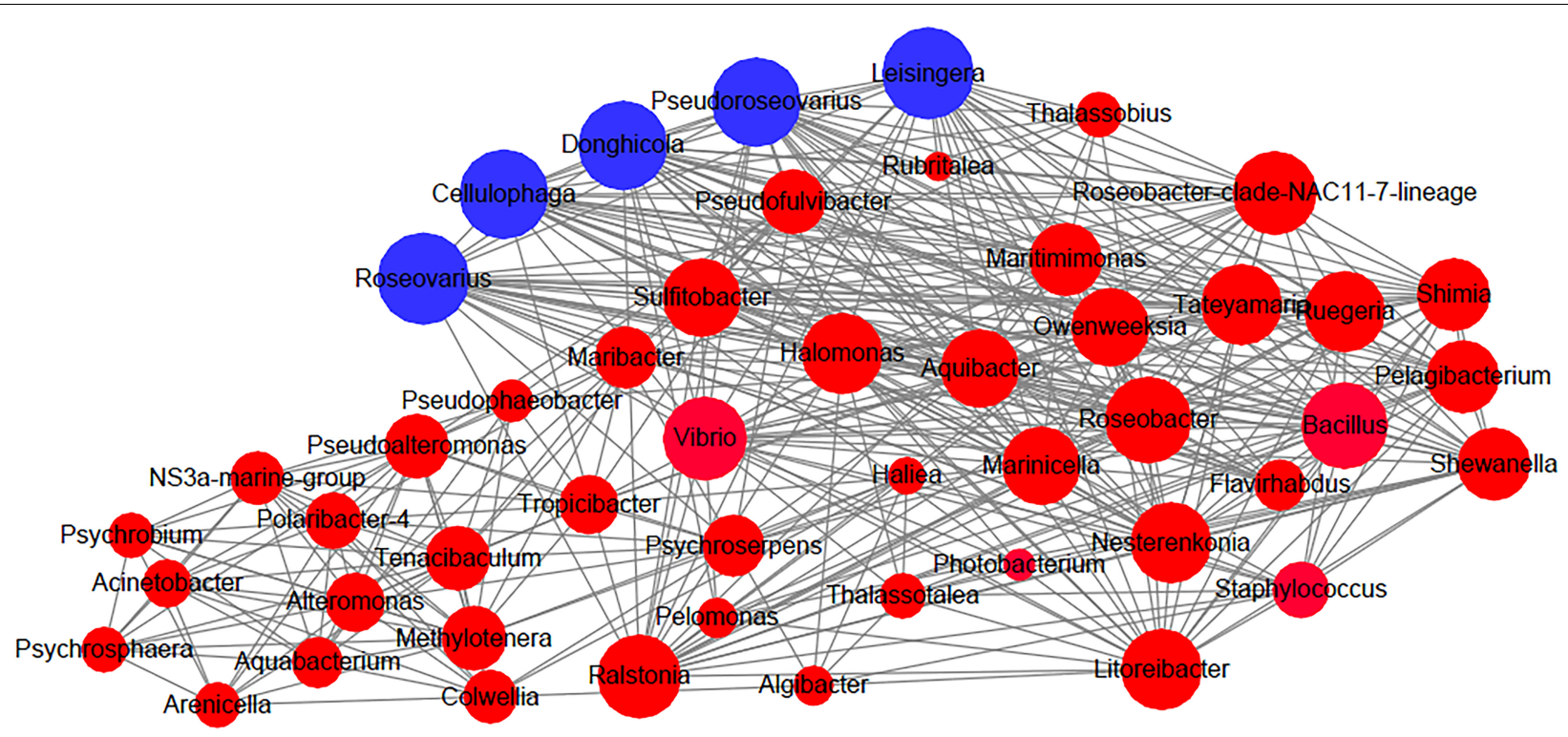

FIGURE 3 | Network analysis for connection among bacterial groups in the intestine of the sea cucumber Apostichopus japonicus during the regeneration process. Note: Blue nodes: the high mean degree taxa. The size of the nodes is proportional to the connected degree.

Within the Proteobacteria, which corresponded to $84.9 \%$ of all the reads in D21 sample, the Gammaproteobacteria (45.8\%) and Alphaproteobacteria (34.5\%) classes prevailed, with Vibrio, unclassified Rhodobacteraceae and Colwellia comprising 35.0, 12.5 , and $3.2 \%$ of the total, respectively. Firmicutes $(62.3 \%)$ were represented mainly by the Bacilli (6.0\%) class, and Staphylococcus (4.0\%) genus. Bacteroidetes was also present (10.2\%), mainly related to the Flavobacteriia (7.8\%) class, and the Sphingobacteriales_norank (2.3\%) genus.

\section{Potential Key Players in the Regenerating Intestinal Microbiome}

Recently, the microbial network has become an increasingly popular tool to analyze microbial community structure. Analyses of microbial networks can help researchers identify high mean degree taxa and further predict keystone species and species interactions. Here, we constructed a co-occurrence network by using all samples during the intestine regeneration process and attempted to identify potential key players during the regeneration period in this study. Roseovarius, Leisingera, Cellulophaga, Pseudoroseovarius, and Donghicola were found to be high mean degree taxa (Figure 3) and may be key players in the intestinal microbial community of $A$. japonicus during the regeneration stages.

\section{Metagenomic Analysis Revealed Functional Differentiation Among the Different Regeneration Stages}

Metagenome sequencing using an Illumina HiSeq platform was performed on intestinal specimens during different regeneration stages. To detect the microbial function response to intestine evisceration and regeneration, 4 time points (the beginning, middle and end of intestinal regeneration, and the CK) were chosen. From the CK and D10, D14, D21 samples, 89,430,618 reads $(13.4 \mathrm{~Gb}), 87,521,564$ reads $(13.1 \mathrm{~Gb}), 92,642,774$ reads $(13.8 \mathrm{~Gb})$, and $88,435,042$ reads $(13.2 \mathrm{~Gb})$, respectively, were obtained. After quality filtering and removing host sequences, 11,089 reads, 11,559 reads, 11,644 reads and 11,558 reads from CK and the D10, D14, D21 stages, respectively, were used for MG-RAST annotation. Bacterial reads occupied 48.3$50.2 \%$, eukaryotic reads occupied $45.4-47.7 \%$, virus reads occupied $2.4-6.2 \%$, and archaeal reads occupied $0.17-0.18 \%$ of all libraries.

The metagenomic genes of samples from different regeneration stages (CK, D10, D14, and D21) were annotated with KEGG orthologous groups. The abundances of functional genes at different levels were calculated, and 238 functional genes were annotated to the metabolic pathway. The pathways of metabolism and human diseases contained the most abundant annotated genes, followed by the pathway of organism system (Supplementary Figure 4). The analysis showed that 23 KEGG functional features in the level 2 were more abundant in one of the samples. Genes annotated to "metabolism of other amino acids," "lipid metabolism," "glycan biosynthesis and metabolism," "metabolism of cofactors and vitamins," "drug resistance: antineoplastic" and others were significantly more abundant in the CK sample; and "excretory system," "folding, sorting, and degradation," "cancers: overview," "cancers: specific types," "immune system," "cell growth and death," and "transport and catabolism" were more abundant in the D10 sample. Regarding D14, genes annotated in "nucleotide metabolism," "digestive system," "metabolism of terpenoids and polyketides" and others were more abundant. For D21, the more abundant genes were annotated in "sensory system," "energy metabolism," and "carbohydrate metabolism" (Figure 4). 


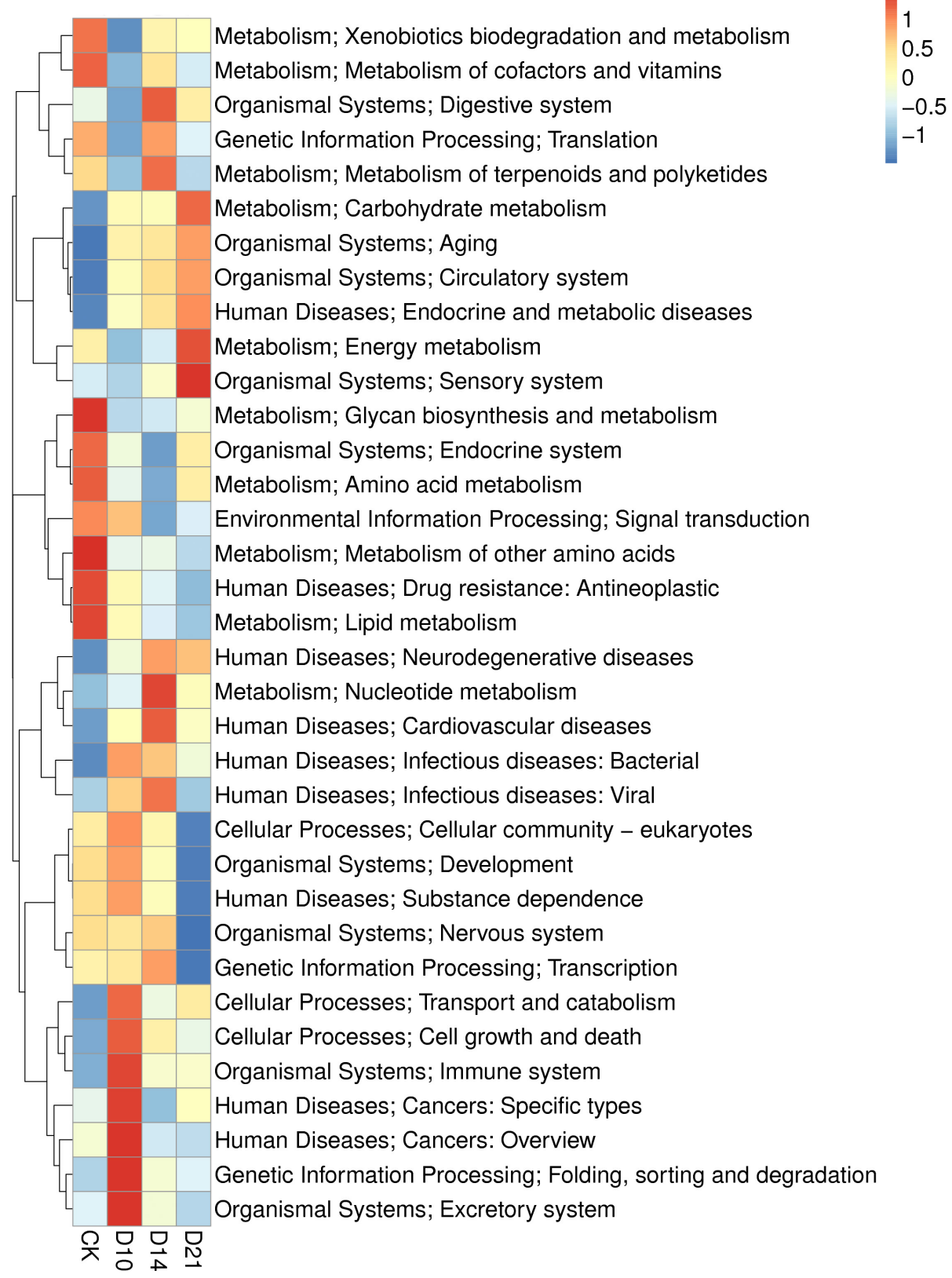

FIGURE 4 | Heatmap of the KEGG analysis of intestinal microbiota in samples from different regeneration stages of the sea cucumber.

In this study, metagenomic data were also compared with the eggNOG database to explore how the microbial gene functions varied in the intestine of $A$. japonicus during intestine regeneration. The functional features of the subsystem category at different levels were analyzed. The genes annotated to "amino acid transport and metabolism" and "replication, recombination and repair" were most abundant, followed by genes annotated to "posttranslational modification, protein turnover, chaperones," "cytoskeleton," and "signal transduction mechanisms" (Supplementary Figure 5). The gene abundance of eggNOG functions also changed during the different regeneration stages. As shown in Figure 5, 16 notable eggNOG functions occurred with a regular pattern during different regeneration stages. In detail, the abundance of genes responsible for homocysteine S-methyltransferase activity, son of sevenless homolog, ubiquitin carboxyl-terminal hydrolase, betalactamase, DNA-dependent RNA polymerase, homocysteine S-methyltransferase activity, heat shock protein, etc. in the level 2 subsystem category were up- or downregulated during the regeneration stages. These gene abundances varied significantly during regeneration stages; however, they tended to revert to the level of the CK specimens in the end stage. 


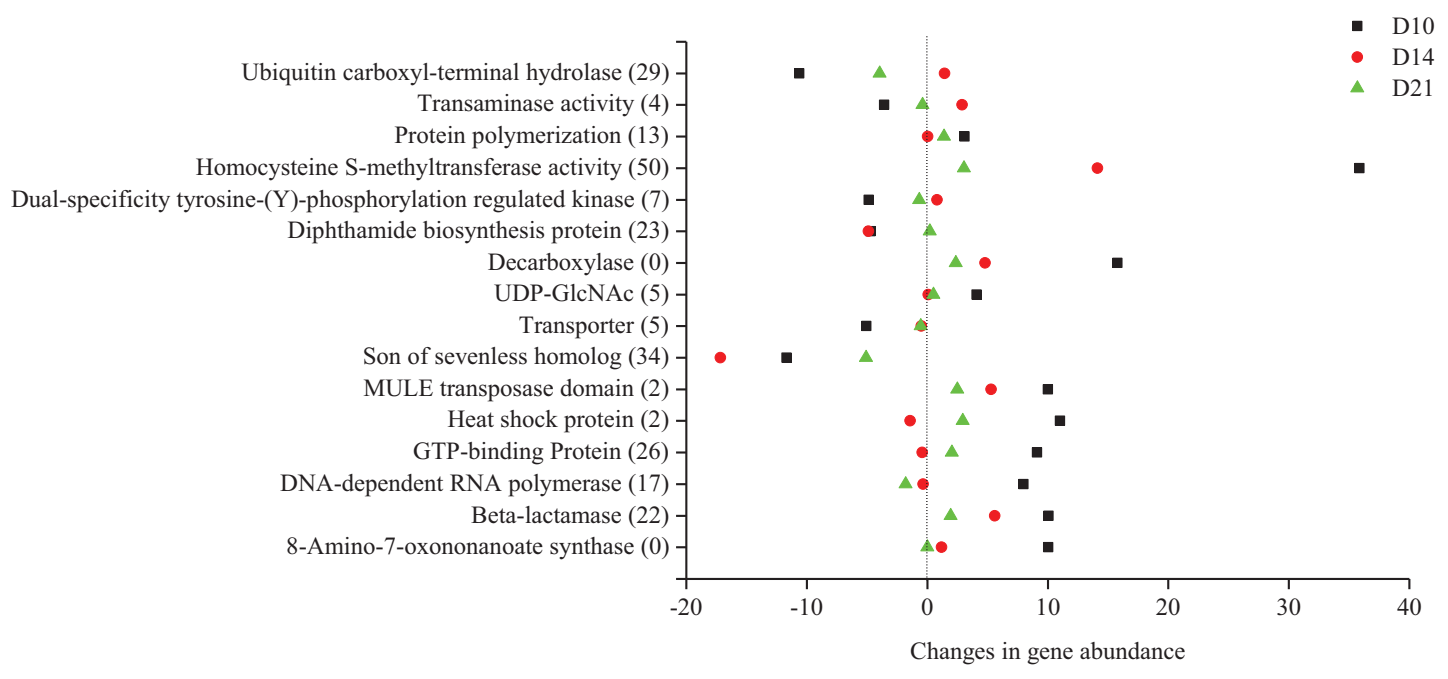

FIGURE 5 | Changes in the functional gene abundance of intestinal microbiota in different regeneration stages.

\section{DISCUSSION}

Intestinal microbiota have been recognized as important players in sea cucumber health and have broad effects on growth and function (Yamazaki et al., 2016; Li et al., 2017a). In recent years, questions concerning how external disturbances affect intestinal microbiota have been addressed, prompted by the development of $16 \mathrm{~S}$ rRNA gene sequencing, powerful metagenomic analyses, and metabolomics technologies. The sea cucumber A. japonicus is an excellent model animal to study in terms of organ regeneration in host-microbiota interactions, however, little is known regarding how the intestinal microbiome composition and function vary in response to intestine regeneration. To the best of our knowledge, the present work is the first analysis of changes in intestinal microbial function during intestine regeneration stages in $A$. japonicus. We demonstrated that intestinal bacterial communities and microbial functional genes in A. japonicus varied significantly with a regular pattern during intestine regeneration stages. Because microbial quantity in the regenerating intestine of $A$. japonicus was decreased, fewer microbial reads were obtained by metagenomics in this study. However, metagenomic analyses can provide a better understanding of the microbiome function response to intestine regeneration events.

In this study, high-throughput sequencing results suggested that bacterial community structures in the intestine of A. japonicus showed significant differences in different regeneration stages. This result is consistent with a previous study (Wang et al., 2018). In the early stage (10 days), a sharp decline in the species richness and diversity of intestinal bacteria was observed, suggesting that the absence of an intestine led to the development of extensive and varied bacterial diversity in the intestine of A. japonicus. However, the diversity of intestinal bacteria then increased gradually. The intestinal bacterial community structures also changed at the phylum and class levels, and particularly at the genus level in the early stage, and then varied significantly in the later stages (14-18 days). Notably, the community composition recovered to a certain extent at the end of regeneration and was similar to the control (Figure 2 and Supplementary Figure 2), which is consistent with a previous report that bacterial community structure in the intestine of A. japonicus tended to be stable after 21 days of regeneration (Wang et al., 2018).

Proteobacteria was the main phylum observed in the intestine of A. japonicus (Gao et al., 2014a; Sha et al., 2016), and Bacteroidetes was the second most abundant phylum (Yang et al., 2015; Yamazaki et al., 2016; Wang et al., 2018) despite the variation in bacterial communities at lower taxonomic levels. In accordance with these previous studies, Proteobacteria was also the most abundant phylum in all samples in this work, particularly in the Gamma- (CK, D10, D14, and D21 samples) and Alphaproteobacteria (D18 sample) classes. Vibrio was the most dominant genus in the CK samples; more than $71.5 \%$ of sequences were assigned to Vibrio. Interestingly, Vibrio was absent in the D10 samples, and its relative abundance gradually increased to $35 \%$ (on average) and returned to the most dominant genus in the D21 samples. Vibrio is widely distributed in marine environments and the digestive tracts of aquatic animals, such as penaeid shrimps (Gomez-Gil et al., 1998) and zebrafish (Brugman et al., 2014). Vibrio was also reported as the predominant genus in the gut of $A$. japonicus in previous studies (Wang et al., 2018; Zhao et al., 2018). Several dozen Vibrio species have been shown to engage in a diversity of beneficial or pathogenic interactions with animal tissue (Ruby et al., 2005), and some species have been used as probiotics in aquaculture (Gao et al., 2014a). A previous study found that zebrafish T lymphocytes can effectively affect the intestinal microbial community structures by suppressing the outgrowth of Vibrio (Brugman et al., 2014). Similarly, the regenerating intestine of $A$. japonicus may present immunoselectivity, and thus the growth of Vibrio may have been suppressed in this study. 
Importantly, the relative abundance of Bacteroidetes was lower in the early regeneration stage but gradually increased to the second most abundant phylum in the D14 and D18 samples and returned to similar levels in the end stage as those observed in the $\mathrm{CK}$ samples. In particular, Flavobacteriaceae_uncultured and Sphingobacteriales_norank prevailed in the D14 and D18 samples, respectively. Previous studies have reported that Bacteroides are specialized in acquiring and digesting a wide variety of polysaccharides (e.g., glycosyl hydrolases, cell-surface carbohydrate-binding proteins, fucoidan, etc.) (Sakai et al., 2002; Xu et al., 2003; Koenig et al., 2011). Flavobacteriaceae are extremely resistant to many antimicrobial agents, and they present low-grade pathogenicity (Jooste and Hugo, 1999). Flavobacteriaceae can produce carotenoids that have antioxidative activities (Shindo et al., 2007) and can utilize fucoidan, fucose, mannose, galactose and glucuronic acid (Sakai et al., 2002). Members of Sphingobacteriales that retain glycoside hydrolase genes can assimilate carbon from glucose and cellulose (Pinnell et al., 2014). The metabolic activities of Bacteroidetes might either directly or indirectly increase the production of carbohydrates, which is beneficial to the host during the intestine regeneration process. Thus, these results further support the notion that intestinal microbiota have regulatory mechanisms to adapt to the regenerating intestine that has an unsound function.

Discovering the keystone taxa, that is, members of the intestinal microbiota that may be important for microbial community structures and integrity, has been a recent focus of researchers interested in understanding intestinal microbial communities in many organisms (Banerjee et al., 2018; Röttjers and Faust, 2018). In the present study, we found that Roseovarius, Leisingera, Cellulophaga, Pseudoroseovarius, and Donghicola were the highly connected taxa in the intestinal microbial community of $A$. japonicus during the regeneration stages. Interestingly, Roseovarius, Leisingera, Pseudoroseovarius, and Donghicola belong to Rhodobacteraceae (within Rhodobacterales), and Cellulophaga belongs to Flavobacteriaceae (within Flavobacteriales). Rhodobacteraceae have been identified as keystone taxa in the microbial community of Nannochloropsis salina in aquatic ecosystems (Geng et al., 2016). Rhodobacteraceae species have also been frequently detected in the intestines of A. japonicus (Gao et al., 2014a; Sha et al., 2016; Wang et al., 2018). Moreover, dietary $\beta$-glucan supplementation can probably modulate the balance of intestinal microbiota and thus promote the proliferation of Rhodobacteraceae in the gut of sea cucumbers and activate the NF-kB signaling pathway (Yang et al., 2015). Yamazaki et al. (2016) found that Rhodobacterales retaining polyhydroxybutyrate (PHB) metabolism genes promoted the growth of $A$. japonicus. In this study, the abundance of Rhodobacterales was increased significantly in samples from D14 to D21 (Supplementary Figure 3). Additionally, 3 OTUs shared by all samples during intestine regeneration were affiliated to Rhodobacterales (Supplementary Table 1); thus, these OTUs might be important for the regrowth of the intestine. We predict that Rhodobacterales and Flavobacteriaceae may function as keystone taxa on the basis of their role in intestine ecosystems and their impact on microbial community structures.
Additionally, Ralstonia (within Burkholderiales), Pelagibacterium, and Aliihoeflea (within Rhizobiales) were also present in all samples during the whole regeneration process. A previous review reported that many members of Rhizobiales and Burkholderiales were consistently identified as the keystone taxa in different ecosystems (Banerjee et al., 2018). They may play an important role in the regenerating intestine of the sea cucumber, and future studies are needed to evaluate this putative role in microbial functions.

Metagenomic data provided additional insight into the dynamics of the regenerating microbiome function. For example, the abundance of Rhodobacterales was increased significantly in the later regeneration stages. At that time, genes annotated to phaA (acetyl-CoA C-acetyltransferase), which is essential in PHB synthesis from acetyl-CoA to PHB (Yamazaki et al., 2016), were correspondingly enriched (data not shown). These results suggest that a transient variation in the intestinal microbiota might have been directly related to the regeneration activity. Another noteworthy observation was that functional features in the subsystem category were changed in a regular pattern. The abundance of functional genes was up- or downregulated clearly during the regeneration process (Figure 5); however, the expression tended to revert to the level of the CK samples in the end stage of regeneration. This pattern was observed in a previous study (Sun L.N. et al., 2013), which reported that the expression level of Wnt6 in the regenerating intestine of the sea cucumber A. japonicus was upregulated at 7 and 14 days and then gradually decreased to the control level at 21 days. These results together suggest that gene expression in the intestine and intestinal microbiota showed the same changing trend in response to intestine regeneration in A. japonicus. In addition, during the regeneration process, functional genes responsible for cell growth and death, carbohydrate metabolism, the digestive system and the immune system were upregulated (Supplementary Figure 6), which is in accord with shifts in specific microbial communities. Together, these results suggest that intestinal microbiota can regulate the abundance of beneficial members and related functional genes, which are helpful for microbial community reconstruction and intestine regrowth in A. japonicus during regeneration. In addition, enriched eukaryotic functional genes reflected high relative levels of fungi in the present study; hereafter, additional experiments should be conducted to further assess how intestinal fungi respond to special life events.

\section{CONCLUSION}

In conclusion, this study revealed that life events can alter the taxonomic and functional features of the intestinal microbiome. We observed large alterations in the abundance of major groups and functional genes; interestingly, these alterations were associated with the intestine regeneration stages. The diversity of the intestinal microbiota was decreased significantly in the early regeneration stage and then increased gradually and recovered in the end stage. The bacterial community composition changed significantly in the regeneration process and tended to recover 
in the end stage. Network analysis revealed that Rhodobacterales and Flavobacteriaceae may function as key players in maintaining the stability of the community structure during the intestine regeneration stages. Metagenomic analysis revealed that the increased abundance of Bacteroidetes elevated the enrichment of genes associated with carbohydrate utilization. The intestinal microbiome was enriched in genes facilitating cell proliferation, digestion, and immunity during the regeneration stages. Some gene expression in the intestine and intestinal microbiota showed the same changing trend in response to intestine regeneration in A. japonicus. Our study indicated that intestinal microbiota have regulatory mechanisms to adapt to the regenerating intestine and provides insights into the host-microbiota interaction response to organ regeneration. The more exact regulatory mechanisms of intestinal microbial composition and functions and their effect on the host remain to be analyzed.

\section{DATA AVAILABILITY}

The datasets generated for this study can be found in sequence read archive, PRJNA512056 and PRJNA518164.

\section{AUTHOR CONTRIBUTIONS}

HZ, QW, SL, JZ, LZ, LS, and HY contributed to the presented idea and design. $\mathrm{HZ}$ implemented the computational and statistical

\section{REFERENCES}

Aronesty, E. (2011). Ea-Utils: Command-Line Tools for Processing Biological Sequencing Data. Available at: http://code.google.com/p/ea-utils (accessed August 16, 2018).

Banerjee, S., Schlaeppi, K., and van der Heijden, M. G. A. (2018). Keystone taxa as drivers of microbiome structure and functioning. Nat. Rev. Microbiol. 16, 567-576. doi: 10.1038/s41579-018-0024-1

Brugman, S., Schneeberger, K., Witte, M., Klein, M. R., Van den Bogert, B., Boekhorst, J., et al. (2014). T lymphocytes control microbial composition by regulating the abundance of Vibrio in the zebrafish gut. Gut Microbes 5 , 737-747. doi: 10.4161/19490976.2014.972228

Buchfink, B., Xie, C., and Huson, D. H. (2015). Fast and sensitive protein alignment using DIAMOND. Nat. Methods 12:59. doi: 10.1038/nmeth.3176

Cantarel, B. L., Coutinho, P. M., Corinne, R., Thomas, B., Vincent, L., and Bernard, H. (2009). The carbohydrate-active enzymes database (CAZy): an expert resource for glycogenomics. Nucleic Acids Res. 37, D233-D238. doi: $10.1093 / \mathrm{nar} / \mathrm{gkn} 663$

Caporaso, J. G., Kuczynski, J., Stombaugh, J., Bittinger, K., Bushman, F. D., Costello, E. K., et al. (2010). QIIME allows analysis of high-throughput community sequencing data. Nat. Methods 7, 335-336. doi: 10.1038/nmeth.f. 303

Cheesman, S. E., Neal, J. T., Mittge, E., Seredick, B. M., and Guillemin, K. (2011). Epithelial cell proliferation in the developing zebrafish intestine is regulated by the Wnt pathway and microbial signaling via Myd88. Proc. Natl. Acad. Sci. U.S.A. 108(Suppl. 1), 4570-4577. doi: 10.1073/pnas.1000072107

Choo, P. S. (2008). "Population status, fisheries and trade of sea cucumbers in Asia," in Sea Cucumbers: a Global Review of Fisheries and Trade, eds V. Toral-Granda, A. Lovatelli, and M. Vasconcellos (Rome: FAO), 81-118.

Dolmatov, I. Y., and Ginanova, T. T. (2009). Post-autotomy regeneration of respiratory trees in the holothurian Apostichopus japonicus (Holothuroidea, Aspidochirotida). Cell Tissue Res. 336, 41-58. doi: 10.1007/s00441-009-0761-6 analyses and took the lead in writing the manuscript. DH assisted with data analysis. LS and HY supervised the findings of this work. All authors provided critical feedback and helped to conduct the research, analysis and manuscript.

\section{FUNDING}

This research was supported by the National Natural Science Foundation of China (No. 41776162), the China Postdoctoral Science Foundation funded project (No. 2018M642712), the Youth Talent Support Program of the Laboratory for Marine Ecology and Environmental Science, the Pilot National Laboratory for Marine Science and Technology (Qingdao; No. LMEES-YTSP-2018-01-09), the Agricultural Seed Project of Shandong Province (No. 2016LZGC032), the Taishan Scholars Program (Distinguished Taishan Scholars), the Key Research Program of the Chinese Academy of Sciences (No. KFJSTS-ZDTP-023), Two-Hundred Talents Plan of Yantai (No. Y839081021), and the Youth Innovation Promotion Association CAS (Nos. 2016196 and 2019209).

\section{SUPPLEMENTARY MATERIAL}

The Supplementary Material for this article can be found online at: https://www.frontiersin.org/articles/10.3389/fmicb. 2019.01165/full\#supplementary-material

Gao, F., Li, F., Tan, J., Yan, J., and Sun, H. (2014a). Bacterial diversity of gut content in sea cucumber (Apostichopus japonicus) and its habitat surface sediment. J. Ocean Univ. China 13, 303-310. doi: 10.1007/s11802-014-2078-7

Gao, F., Li, F., Tan, J., Yan, J., and Sun, H. (2014b). Bacterial community composition in the gut content and ambient sediment of sea cucumber Apostichopus japonicus revealed by $16 \mathrm{~S}$ rRNA gene pyrosequencing. PLoS One 9:e100092. doi: 10.1371/journal.pone.0100092

García-Arrarás, J. E., Estrada-Rodgers, L., Santiago, R., Torres, I. I., DíazMiranda, L., and Torres-Avillán, I. (1998). Cellular mechanisms of intestine regeneration in the sea cucumber, Holothuria glaberrima Selenka (Holothuroidea: Echinodermata). J. Exp. Zool. 281, 288-304. doi: 10.1002/(sici)1097-010x(19980701)281:4<288::aid-jez5>3.0.co;2-k

Geng, H., Tran-Gyamfi, M. B., Lane, T. W., Sale, K. L., and Yu, E. T. (2016). Changes in the structure of the microbial community associated with nannochloropsis salina following treatments with antibiotics and bioactive compounds. Front. Microbiol. 7:01155. doi: 10.3389/fmicb.2016.01155

Gomez-Gil, B., Tron-Mayén, L., Roque, A., Turnbull, J. F., Inglis, V., and Guerra-Flores, A. L. (1998). Species of Vibrio isolated from hepatopancreas, haemolymph and digestive tract of a population of healthy juvenile Penaeus vannamei. Aquaculture 163, 1-9. doi: 10.1016/S0044-8486(98) 00162-8

Jooste, P. J., and Hugo, C. J. (1999). The taxonomy, ecology and cultivation of bacterial genera belonging to the family Flavobacteriaceae. Int. J. Food Microbiol. 53, 81-94. doi: 10.1016/s0168-1605(99)00162-2

Knoop, K. A., Gustafsson, J. K., Mcdonald, K. G., Kulkarni, D. H., Coughlin, P. E., Mccrate, S., et al. (2017). Microbial antigen encounter during a preweaning interval is critical for tolerance to gut bacteria. Sci. Immunol. 2:eaao1314. doi: 10.1126/sciimmunol.aao1314

Koenig, J. E., Spor, A., Scalfone, N., Fricker, A. D., Stombaugh, J., Knight, R., et al. (2011). Succession of microbial consortia in the developing infant gut microbiome. Proc. Natl. Acad. Sci. U.S.A. 108(Suppl. 1), 4578-4585. doi: 10. 1073/pnas.1000081107 
Langmead, B., and Salzberg, S. L. (2012). Fast gapped-read alignment with Bowtie 2. Nat. Methods 9, 357-359. doi: 10.1038/nmeth.1923

Li, C., Ren, Y., Jiang, S., Zhou, S., Zhao, J., Wang, R., et al. (2017a). Effects of dietary supplementation of four strains of lactic acid bacteria on growth, immune-related response and genes expression of the juvenile sea cucumber Apostichopus japonicus Selenka. Fish Shellfish Immunol. 74, 69-75. doi: 10.1016/ j.fsi.2017.12.037

Li, X., Sun, L., Yang, H., Zhang, L., Miao, T., Xing, L., et al. (2017b). Identification and expression characterization of WntA during intestinal regeneration in the sea cucumber Apostichopus japonicus. Comp. Biochem. Physiol. B Biochem. Mol. Biol. 210, 55-63. doi: 10.1016/j.cbpb.2017.06.005

Li, W., and Godzik, A. (2006). Cd-hit: a fast program for clustering and comparing large sets of protein or nucleotide sequences. Bioinformatics 22, 1658-1659. doi: 10.1093/bioinformatics/btl158

Luo, R., Liu, B., Xie, Y., Li, Z., Huang, W., Yuan, J., et al. (2012). SOAPdenovo2: an empirically improved memory-efficient short-read de novo assembler. Gigascience 1, 18-18. doi: 10.1186/2047-217X-1-18

Ma, Y. X., Li, L. Y., Li, M., Chen, W., Bao, P. Y., Yu, Z. C., et al. (2019). Effects of dietary probiotic yeast on growth parameters in juvenile sea cucumber, Apostichopus japonicus. Aquaculture 499, 203-211. doi: 10.1016/j.fsi.2012. 10.005

Minoru, K., Susumu, G., Masahiro, H., Aoki-Kinoshita, K. F., Masumi, I., Shuichi, K., et al. (2006). From genomics to chemical genomics: new developments in KEGG. Nucleic Acids Res. 34, D354-D357. doi: 10.1093/nar/ gkj102

Nielsen, H. B., Almeida, M., Juncker, A. S., Rasmussen, S., Li, J., Sunagawa, S., et al. (2014). Identification and assembly of genomes and genetic elements in complex metagenomic samples without using reference genomes. Nat. Biotechnol. 32, 822-828. doi: 10.1038/nbt.2939

Okada, M., Bothin, C., Kanazawa, K., and Midtvedt, T. (1999). Experimental study of the influence of intestinal flora on the healing of intestinal anastomoses. $\mathrm{Br}$. J. Surg. 86, 961-965. doi: 10.1046/j.1365-2168.1999.01161.x

Pinnell, L. J., Dunford, E., Ronan, P., Hausner, M., and Neufeld, J. D. (2014). Recovering glycoside hydrolase genes from active tundra cellulolytic bacteria. Can. J. Microbiol. 60, 469-476. doi: 10.1139/cjm-2014-0193

Qi, W., Zhang, X., Chen, M., Li, W., and Zhang, P. (2017). Comparison of intestinal microbiota and activities of digestive and immune-related enzymes of sea cucumber Apostichopus japonicus in two habitats. Chin. J. Oceanol. Limnol. 36, 1-12. doi: $10.1007 / \mathrm{s} 00343-018-7075-\mathrm{z}$

Qin, J., Li, R., Raes, J., Arumugam, M., Burgdorf, K. S., Manichanh, C., et al. (2010). A human gut microbial gene catalogue established by metagenomic sequencing. Nature 464, 59-65. doi: 10.1038/nature08821

Röttjers, L., and Faust, K. (2018). From hairballs to hypotheses-biological insights from microbial networks. FEMS Microbiol. Rev. 42, 761-780. doi: 10.1093/ femsre/fuy030

Ruby, E. G., Urbanowski, M., Campbell, J., Dunn, A., Faini, M., Gunsalus, R., et al. (2005). Complete genome sequence of Vibrio fischeri: a symbiotic bacterium with pathogenic congeners. Proc. Natl. Acad. Sci. U.S.A. 102, 3004-3009. doi: 10.1073/pnas.0409900102

Sakai, T., Kimura, H., and Kato, I. (2002). A marine strain of Flavobacteriaceae utilizes brown seaweed fucoidan. Mar. Biotechnol. 4, 399-405. doi: 10.1007/ s10126-002-0032-y

Sean, P., Kristoffer, F., Damian, S., Kalliopi, T., Alexander, R., Jaime, H. C., et al. (2014). eggNOG v4.0: nested orthology inference across 3686 organisms. Nucleic Acids Res. 42, D231-D239. doi: 10.1093/nar/gkt1253

Sha, Y., Liu, M., Wang, B., Jiang, K., Sun, G., and Wang, L. (2016). Gut bacterial diversity of farmed sea cucumbers Apostichopus japonicus with different growth rates. Microbiology 85, 109-115. doi: 10.1134/s0026261716010112

Sharon, G., Sampson, T. R., Geschwind, D. H., and Mazmanian, S. K. (2016). The central nervous system and the gut microbiome. Cell 167, 915-932. doi: 10.1016/j.cell.2016.10.027

Shindo, K., Kikuta, K., Suzuki, A., Katsuta, A., Kasai, H., Yasumoto-Hirose, M., et al. (2007). Rare carotenoids, (3R)-saproxanthin and (3R, 2'S)-myxol, isolated from novel marine bacteria (Flavobacteriaceae) and their antioxidative activities. Appl. Microbiol. Biotechnol. 74, 1350-1357. doi: 10.1007/s00253-0060774-y

Shukalyuk, A. I., and Dolmatov, I. Y. (2001). Regeneration of the digestive tube in the holothurian Apostichopus japonicus after evisceration. Russ. J. Mar. Biol. 27, 168-173. doi: 10.1007/s00441-009-0761-6
Sun, L., Chen, M., Yang, H., Wang, T., Liu, B., Shu, C., et al. (2011). Large scale gene expression profiling during intestine and body wall regeneration in the sea cucumber Apostichopus japonicus. Comp. Biochem. Physiol. Part D Genomics Proteomics 6, 195-205. doi: 10.1016/j.cbd.2011.03.002

Sun, L., Lin, C., Li, X., Xing, L., Huo, D., Sun, J., et al. (2018). Comparative phosphoand acetyl proteomics analysis of posttranslational modifications regulating intestine regeneration in sea cucumbers. Front. Physiol. 9:836. doi: 10.3389/ fphys.2018.00836

Sun, L., Sun, J., Li, X., Zhang, L., Yang, H., and Wang, Q. (2017a). Understanding regulation of microRNAs on intestine regeneration in the sea cucumber Apostichopus japonicus using high-throughput sequencing. Comp. Biochem. Physiol. Part D Genomics Proteomics 22, 1-9. doi: 10.1016/j.cbd.2017.01.001

Sun, L., Xu, D., Xu, Q., Sun, J., Xing, L., Zhang, L., et al. (2017b). iTRAQ reveals proteomic changes during intestine regeneration in the sea cucumber Apostichopus japonicus. Comp. Biochem. Physiol. Part D Genomics Proteomics 22, 39-49. doi: 10.1016/j.cbd.2017.02.004

Sun, L., Yang, H., Chen, M., Ma, D., and Lin, C. (2013). RNA-seq reveals dynamic changes of gene expression in key stages of intestine regeneration in the sea cucumber Apostichopus japonicus. PLoS One 8:e69441. doi: 10.1371/journal. pone. 0069441

Sun, L. N., Yang, H. S., Chen, M. Y., and Xu, D. X. (2013). Cloning and expression analysis of Wnt6 and Hox6 during intestinal regeneration in the sea cucumber Apostichopus japonicus. Genet. Mol. Res 12, 5321-5334. doi: 10.4238/ 2013

Wang, L., Li, X., Hu, D., Lai, Q., and Shao, Z. (2015). Tenacibaculum holothuriorum sp. nov., isolated from the sea cucumber Apostichopus japonicus intestine. Int. J. Syst. Evol. Microbiol. 65:4347. doi: 10.1099/ijsem.0.000574

Wang, L., Zhao, X., Xu, H., Bao, X., Liu, X., Chang, Y., et al. (2018). Characterization of the bacterial community in different parts of the gut of sea cucumber (Apostichopus Japonicus) and its variation during gut regeneration. Aquac. Res. 49, 1987-1996. doi: 10.1111/are.13654

Wang, X., Sun, Y., Wang, L., Li, X., Qu, K., and Xu, Y. (2017). Synbiotic dietary supplement affects growth, immune responses and intestinal microbiota of Apostichopus japonicas. Fish Shellfish Immunol. 68, 232-242. doi: 10.1016/j.fsi. 2017.07.027

Xu, J., Bjursell, M. K., Himrod, J., Deng, S., Carmichael, L. K., Chiang, H. C., et al. (2003). A genomic view of the human-Bacteroides thetaiotaomicron symbiosis. Science 299, 2074-2076. doi: 10.1126/science.1080029

Yamazaki, Y., Meirelles, P. M., Mino, S., Suda, W., Oshima, K., Hattori, M., et al. (2016). Individual Apostichopus japonicus fecal microbiome reveals a link with polyhydroxybutyrate producers in host growth gaps. Sci. Rep. 6:21631. doi: $10.1038 /$ srep21631

Yang, G., Xu, Z., Tian, X., Dong, S., and Peng, M. (2015). Intestinal microbiota and immune related genes in sea cucumber (Apostichopus japonicus) response to dietary $\beta$-glucan supplementation. Biochem. Biophys. Res. Commun. 458 98-103. doi: 10.1016/j.bbrc.2015.01.074

Zhang, X., Nakahara, T., Murase, S., Nakata, H., Inoue, T., and Kudo, T. (2013). Physiological characterization of aerobic culturable bacteria in the intestine of the sea cucumber Apostichopus japonicus. J. Gen. Appl. Microbiol. 59, 1-10. doi: $10.2323 /$ jgam. 59.1

Zhang, X., Sun, L., Yuan, J., Sun, Y., Gao, Y., Zhang, L., et al. (2017). The sea cucumber genome provides insights into morphological evolution and visceral regeneration. PLoS Biol. 15:e2003790. doi: 10.1371/journal.pbio.2003790

Zhao, Y., Lei, Y., Wan, J., Sun, H., Wang, Y., and Qin, Z. (2018). Effects of a potential autochthonous probiotic Bacillus subtilis 2-1 on the growth and intestinal microbiota of juvenile sea cucumber, Apostichopus japonicus Selenka. J. Ocean Univ. China 17, 363-370. doi: 10.1007/s11802-018-3402-4

Conflict of Interest Statement: The authors declare that the research was conducted in the absence of any commercial or financial relationships that could be construed as a potential conflict of interest.

Copyright (๑ 2019 Zhang, Wang, Liu, Huo, Zhao, Zhang, Zhao, Sun and Yang. This is an open-access article distributed under the terms of the Creative Commons Attribution License (CC BY). The use, distribution or reproduction in other forums is permitted, provided the original author $(s)$ and the copyright owner(s) are credited and that the original publication in this journal is cited, in accordance with accepted academic practice. No use, distribution or reproduction is permitted which does not comply with these terms. 